\title{
Gauge Models in Modified Triplectic Quantization
}

\author{
B. Geyer ${ }^{a, b}$, P.M. Lavrov ${ }^{a, c,}$ 色and P.Yu. Moshin ${ }^{c}$ \\ a) Center of Theoretical Studies, Leipzig University, \\ Augustusplatz 10/11, 04109 Leipzig, Germany \\ $\left.{ }^{b}\right)$ Instituto de Fisica, Universidade de São Paulo, \\ Caixa Postal 66318-CEP, 05315-970 São Paulo, SP, Brazil \\ c) Tomsk State Pedagogical University, 634041 Tomsk, Russia
}

\begin{abstract}
The modified triplectic quantization is applied to several well-known gauge models: the Freedman-Townsend model of non-abelian antisymmetric tensor fields, $W_{2}$-gravity, and 2D gravity with dynamical torsion. For these models we obtain explicit solutions of those generating equations that determine the quantum action and the gauge-fixing functional. Using these solutions, we construct the vacuum functional, determine the $S p(2)$-invariant effective actions and obtain the corresponding transformations of extended BRST symmetry.
\end{abstract}

\section{Introduction}

In recent years the development of covariant quantization rules for general gauge theories on the basis of extended BRST symmetry has become increasingly popular [1] - [13].

The realization of the principle of extended BRST symmetry, combining BRST [14 and anti-BRST [15] transformations, naturally unifies the treatment of auxiliary variables that serve to parametrize the gauge in the functional integral and those that enter the quantum action determined by the corresponding generating equations. Basically, the above tendency manifests itself in enlarging the configuration space of the quantum action with auxiliary gauge-fixing variables (see, e.g., Refs. [1, 2, 3]). Recently, however, it has been strengthened by extending the concept of generating equations to cover the case of introducing the gauge [2, 6].

The method of $S p(2)$-covariant quantization [四] was one of the first to provide a realization of extended BRST symmetry for general gauge theories, i.e., theories of any stage of reducibility with a closed or open algebra of gauge transformations. The complete configuration space $\phi^{A}$ of a gauge theory, considered in this approach, is constructed by the rules of the BV quantization [16 and consists of the initial classical fields supplemented by the pyramids of auxiliary variables, i.e., ghosts, antighosts and Lagrange multipliers, according to the corresponding stage of reducibility. Even though these auxiliary variables originally [16 play different roles in the construction of the quantum theory, their consideration within the $S p(2)$-covariant formalism allows to achieve a remarkable

\footnotetext{
${ }^{1}$ E-mail: geyer@itp.uni-leipzig.de

${ }^{2}$ E-mail: lavrov@tspu.edu.ru
} 
uniformity of description. Namely, in the framework of the $S p(2)$-covariant approach, the pyramids of ghosts are combined with the corresponding pyramids of antighosts and Lagrange multipliers into irreducible representations of the group $S p(2)$, which form completely symmetric $S p(2)$-tensors and enter the quantum theory on equal footing in terms of both the quantum action and the gauge fixing functional. The quantum action of the $S p(2)$-covariant formalism depends on an extended set of variables, including, besides the fields $\phi^{A}$, also the sets of antifields $\phi_{A a}^{*}$ and $\bar{\phi}_{A}$. In the case of linear dependence of the quantum action on $\phi_{A a}^{*}$ and $\bar{\phi}_{A}$ they may be interpreted as sources of extended BRST transformations and sources of mixed BRST and anti-BRST transformations, respectively.

In [3], a consistent superfield formulation of the $S p(2)$-covariant rules was proposed. This approach allows to combine all the variables of the $S p(2)$-covariant formalism, namely, the fields and antifields $\left(\phi^{A}, \phi_{A a}^{*}, \bar{\phi}_{A}\right)$ that enter the quantum action, the auxiliary variables $\left(\pi^{A a}, \lambda^{A}\right)$ that serve to parametrize the gauge, and, finally, the sources $J_{A}$ to the fields $\phi^{A}$, into superfields $\Phi^{A}(\theta)=\phi^{A}+\pi^{A a} \theta_{a}+\frac{1}{2} \lambda^{A} \theta_{a} \theta^{a}$ and superantifields (supersources) $\bar{\Phi}_{A}(\theta)=\bar{\phi}_{A}-\theta^{a} \phi_{A a}^{*}-\frac{1}{2} \theta_{a} \theta^{a} J_{A}$, defined on a superspace with two scalar Grassmann coordinates $\theta_{a}$. The quantum action of this theory is defined as a functional of superfields and superantifields, $S(\Phi, \bar{\Phi})$, which makes it possible to realize the transformations of extended BRST symmetry in terms of supertranslations along the Grassmann coordinates.

Moreover, in the recent paper [四] the superspace approach was extended by considering not only the (sub)group of translations but also the full group of conformal transformations on the superspace with two Grassmann coordinates. The generators of this conformal group span an algebra isomorphic to the superalgebra $s l(1,2)$. In this approach it is possible to consider massive gauge theories by introducing mass-dependent BRST and antiBRST operators that are related to translations coupled (with the factor $m^{2}$ ) to special conformal transformations. Furthermore, the transformations of $S p(2)$-symmetry, including the symmetries which underly the conservation of the ghost number and the "new ghost number", are realized as (symplectic) rotations and dilatations, respectively.

In the framework of the triplectic quantization [2], another modification of the $S p(2)$ covariant approach was proposed, based on a different extension of the configuration space of the quantum action. Namely, it was suggested to consider the auxiliary fields $\pi^{A a}$ as variables anticanonically conjugated to the antifields $\bar{\phi}_{A}$ with the corresponding redefinition of the extended antibrackets [1] which appear in the generating equations for the quantum action. Another feature of the triplectic formalism is that the gauge-fixing part of the action in the functional integral is determined by generating equations formally similar to the equations that describe the quantum action. The entire set of variables necessary for the construction of the vacuum functional in the triplectic formalism coincides with the corresponding set of the $S p(2)$-covariant approach and is composed by the fields $\left(\phi^{A}, \phi_{A a}^{*}\right)$ and $\left(\pi^{A a}, \bar{\phi}_{A}\right)$ anticanonically conjugated to each other in the sense of modified antibrackets, as well as by the remaining auxiliary fields $\lambda^{A}$ that serve to parametrize the gauge-fixing functional.

In the recent paper [6], a modification of the triplectic formalism was proposed, whose essential ingredients we will now briefly review.

While retaining the space of variables of the triplectic formalism and accepting the idea of imposing generating equations on both the quantum action and the gauge-fixing functional, the formalism [6] modifies the system of these equations, as well as the definition of the vacuum functional, in order to ensure the correct boundary condition for the quantum action,

$$
\left.W\right|_{\Phi^{*}=\bar{\phi}=\hbar=0}=S_{\mathrm{cl}},
$$

which explicitly allows to take into account the information contained in the classical action. It also implies that the classical action of a theory satisfies (in the limit $\hbar \rightarrow 0$ ) 
the generating equations for the quantum action $W=W\left(\phi, \phi^{*}, \pi, \bar{\phi}\right)$,

$$
\frac{1}{2}(W, W)^{a}+V^{a} W=i \hbar \Delta^{a} W
$$

in complete analogy with earlier quantization schemes, and in contrast to the original triplectic formalism [2]. The gauge-fixing functional $X=X\left(\phi, \phi^{*}, \pi, \bar{\phi} ; \lambda\right)$ of the modified triplectic formalism satisfies similar generating equations,

$$
\frac{1}{2}(X, X)^{a}-U^{a} X=i \hbar \Delta^{a} X
$$

The above generating equations are expressed in terms of the extended antibrackets

$$
(F, G)^{a}=\left(\frac{\delta F}{\delta \phi^{A}} \frac{\delta G}{\delta \phi_{A a}^{*}}+\varepsilon^{a b} \frac{\delta F}{\delta \pi^{A a}} \frac{\delta G}{\delta \bar{\phi}_{A}}\right)-(F \leftrightarrow G)(-1)^{(\varepsilon(F)+1)(\varepsilon(G)+1)}
$$

and the differential operators

$$
\begin{aligned}
\Delta^{a} & =(-1)^{\varepsilon_{A}} \frac{\delta_{l}}{\delta \phi^{A}} \frac{\delta}{\delta \phi_{A a}^{*}}+(-1)^{\varepsilon_{A}+1} \varepsilon^{a b} \frac{\delta_{l}}{\delta \pi^{A b}} \frac{\delta}{\delta \bar{\phi}_{A}}, \\
V^{a} & =\varepsilon^{a b} \phi_{A b}^{*} \frac{\delta}{\delta \bar{\phi}_{A}}, \quad U^{a}=(-1)^{\varepsilon_{A}+1} \pi^{A a} \frac{\delta_{l}}{\delta \phi^{A}},
\end{aligned}
$$

where the derivatives with respect to the antifields are taken from the left, and $\varepsilon^{a b}$ is the antisymmetric tensor with the normalization $\varepsilon^{12}=1$. The operators $V^{a}$ and $U^{a}$ are closely related to the differential operators which were introduced earlier in the framework of the superfield formalism [3], and which have a clear geometrical meaning as generators of supertranslations in superspace.

Given the quantum action $W$ and the gauge-fixing functional $X$, the vacuum functional $Z \equiv Z(J=0)$ in the framework of the modified triplectic quantization [6] is defined by

$$
Z=\int d \phi d \phi^{*} d \pi d \bar{\phi} d \lambda \exp \left\{\frac{i}{\hbar}\left(W+X+S_{0}\right)\right\}, \quad S_{0}=\phi_{A a}^{*} \pi^{A a} .
$$

Let us note that choosing the gauge fixing functional, $X=X(\phi, \pi, \bar{\phi} ; \lambda)$, in the form

$$
X=\left(\bar{\phi}_{A}-\frac{\delta F}{\delta \phi^{A}}\right) \lambda^{A}-\frac{1}{2} \epsilon_{a b} U^{a} U^{b} F, \quad F=F(\phi),
$$

solves eqs. (1.2) (with $\Delta^{a} X$ being identical zero). Then, the integrand in eq. (1.4) is invariant under the following transformations (cf., Ref. [6])

$$
\begin{aligned}
\delta \phi^{A a} & =-\left(\frac{\delta W}{\delta \phi_{A a}^{*}}-\pi^{A a}\right) \mu_{a} \\
\delta \phi_{A a}^{*} & =\mu_{a}\left(\frac{\delta W}{\delta \phi^{A}}+\frac{\delta^{2} F}{\delta \phi^{A} \delta \phi^{B}} \lambda^{B}+\left(-1^{\varepsilon_{A}} \frac{1}{2} \pi^{B b} \frac{\delta^{3} F}{\delta \phi^{A} \delta \phi^{B} \delta \phi^{C}} \pi^{C c}\right)\right. \\
\delta \pi^{A a} & =\varepsilon^{a b}\left(\frac{\delta W}{\delta \bar{\phi}_{A}}-\lambda^{A}\right) \mu_{b} \\
\delta \bar{\phi}_{A} & =\mu_{a} \varepsilon^{a b}\left(\frac{\delta W}{\delta \pi^{A b}}+\phi_{A b}^{*}\right)+\mu_{a} \frac{\delta^{2} F}{\delta \phi^{A} \delta \phi^{B}} \pi^{B a} \\
\delta \lambda^{A} & =0
\end{aligned}
$$


Here, $\mu_{a}$ is a doublet of constant anticommuting parameters. If, in addition, $W=$ $W\left(\phi, \phi^{*}, \bar{\phi}\right)$ is assumed not to depend on $\pi^{A a}$ then, obviously, the master equations (1.1) and the vacuum functional (1.4) are reduced to those of the $S p(2)$-covariant formalism.

The aim of this paper is to apply the above prescriptions of the modified triplectic formalism for quantizing several gauge models.

In Section 2, we consider the model of an antisymmetric tensor field suggested by Freedman and Townsend [17]. The Freedman-Townsend (FT) model is an abelian gauge theory of first stage reducibility. The corresponding complete configuration space is constructed by the rules of the $S p(2)$-covariant formalism [1] for reducible gauge theories. In the case of the FT model, the generating equations (1.1) that determine the quantum action in the framework of the modified triplectic formalism can be solved exactly, which allows one to obtain the exact form of the vacuum functional in terms of the effective $S p(2)$-invariant action $S_{\text {eff }}$ as well as the corresponding transformations of extended BRST symmetry.

In Sections 3 and 4, we consider the gauge model of $W_{2}$-gravity [18] and the theory of two-dimensional gravity with dynamical torsion [19], respectively. Both these models are examples of irreducible gauge theories with a closed algebra, and their configuration spaces are constructed by the rules of the $S p(2)$-covariant quantization for irreducible theories. In order to obtain closed solutions of the above generating equations that determine the quantum action in the case of these gauge models, one has to introduce some regularization for $\Delta^{a} W$ when it occurs to be proportional to $\delta(0)$. Unfortunately, the regularizations which reduces all terms containing $\delta(0)$ to zero (see, e.g., [1]) cannot be used here since both models under consideration are strictly two-dimensional and, therefore, it is not possible to use dimensional regularization on which that procedure is based. Instead, one could use Pauli-Villars regularization as has been proposed in Ref. [20] and applied in Refs. 21] and 22 to the case of $W_{2}$ and $W_{3}$-gravity, respectively. As an intermediate step we consider here only the tree approximation, i.e., we determine explicit solutions of the classical master equations. With them we obtain a closed form of the vacuum functional and the corresponding transformations of extended BRST symmetry as well as the related effective action, $S_{\text {eff }}$, which depends on the fields only.

\section{Freedman-Townsend Model}

In the first order formalism, the theory of a non-abelian antisymmetric field $H_{\mu \nu}^{p}$, suggested by Freedman and Townsend [17], is described by the action 3 ]

$$
S_{c l}\left(A_{\mu}^{p}, H_{\mu \nu}^{p}\right)=\int d^{4} x\left(-\frac{1}{4} \varepsilon^{\mu \nu \rho \sigma} F_{\mu \nu}^{p} H_{\rho \sigma}^{p}+\frac{1}{2} A_{\mu}^{p} A^{p \mu}\right)
$$

where $A_{\mu}^{p}$ is an (auxiliary) gauge field with the strength $F_{\mu \nu}^{p}=\partial_{\mu} A_{\nu}^{p}-\partial_{\nu} A_{\mu}^{p}+f^{p q r} A_{\mu}^{q} A_{\nu}^{r}$ (the coupling constant is absorbed into the structure coefficients $f^{p q r}$ ), and the LeviCivita tensor $\varepsilon^{\mu \nu \rho \sigma}$ is normalized as $\varepsilon^{0123}=1$. Eliminating the auxiliary field $A_{\mu}^{p}$ with the help of the field equations leads to the more complicated action of the second order formalism [17].

The action (2.1) is invariant under the gauge transformations

$$
\delta A_{\mu}^{p}=0, \quad \delta H_{\mu \nu}^{p}=\mathcal{D}_{\mu}^{p q} \xi_{\nu}^{q}-\mathcal{D}_{\nu}^{p q} \xi_{\mu}^{q} \equiv \mathcal{R}_{\mu \nu \alpha}^{p q} \xi^{q \alpha},
$$

where $\xi_{\mu}^{p}$ are arbitrary parameters, and $\mathcal{D}_{\mu}^{p q}$ is the covariant derivative corresponding to the gauge field $A_{\mu}^{p}\left(\mathcal{D}_{\mu}^{p q}=\delta^{p q} \partial_{\mu}+f^{p r q} A_{\mu}^{r}\right)$.

\footnotetext{
${ }^{3}$ Contrary to the original notations [17], we denote the antisymmetric tensor field by $H$ in order to avoid confusion with the auxiliary fields to be introduced below.
} 
The gauge transformations (2.2) form an abelian algebra with the generators $R_{\mu \nu \alpha}^{p q}$. These gauge transformations are not all independent, namely, for $\xi_{\nu}^{p}=\mathcal{D}_{\nu}^{q p} \xi^{p}$ they vanish on-shell. Therefore, at the extremals of the action (2.1) the generators $\mathcal{R}_{\mu \nu \alpha}^{p q}$ have zero modes $\mathcal{Z}_{\mu}^{p q} \equiv \mathcal{D}_{\mu}^{p q}$,

$$
\mathcal{R}_{1 \mu \nu}^{p q} \equiv \mathcal{R}_{\mu \nu \alpha}^{p r} \mathcal{Z}^{r q \alpha}=\varepsilon_{\mu \nu \alpha \beta} f^{p r q} \frac{\delta S_{\mathrm{cl}}}{\delta H_{\alpha \beta}^{r}},
$$

which, in their turn, are linearly independent. According to the generally accepted terminology, the model (2.1), (2.2) and (2.3) is an abelian gauge theory of first stage reducibility.

Note that the gauge structure of the FT model [17] is similar to that of the Witten string [23]. The FT model also has been proved to be a convenient conceptual laboratory for the study of the $S$-matrix unitarity in the framework of covariant quantization [27]. There, it was shown that the application of the BV quantization rules to the model leads to a physically unitary theory being equivalent to a non-linear $\sigma$-model in $d=4$ dimensions [17. Note also that various aspects of the quantization of the FT model in the framework of standard BRST symmetry have been discussed in Refs. [24, 28].

Now let us consider the reducible gauge model (2.1), (2.2) and (2.3) in the framework of the modified triplectic quantization.

To this end, we first introduce the complete configuration space $\phi^{A}$, which is constructed according to the standard prescriptions of the $S p(2)$-covariant formalism [1] for reducible gauge theories. Namely, the space of the variables $\phi^{A}$ consists of the initial classical fields $A^{p \mu}$ and $H^{p \mu \nu}$, supplemented, firstly, by $S p(2)$-doublets of Faddeev-Popov ghosts, $C_{\mu}^{p a}$, introduced according to the gauge parameters $\xi_{\mu}^{p}$ in eq. (2.2); secondly, by additional sets of first-stage ghost fields, $C^{p a b}$, being symmetric $S p(2)$ tensors, introduced according to the gauge parameters $\xi^{p}$ for the generators $R_{1 \mu \nu}^{p q}$ in eq. (2.3); and, finally, by sets of auxiliary fields (Lagrange multipliers) $B_{\mu}^{p}$, corresponding to the gauge parameters $\xi_{\mu}^{p}$, and first-stage $S p(2)$-doublets $B^{p a}$, corresponding to the parameters $\xi^{p}$.

The fields $\phi^{A}$ of the complete configuration space take values in the adjoint representation of a non-abelian gauge group:f

$$
\phi^{A}=\left(A^{\mu}, H^{\mu \nu} ; B^{\mu}, B^{a} ; C^{\mu a}, C^{a b}\right) .
$$

The Grassmann parities of the fields $\phi^{A}$ are given by

$$
\varepsilon\left(A^{\mu}\right)=\varepsilon\left(H^{\mu \nu}\right)=\varepsilon\left(B^{\mu}\right)=\varepsilon\left(C^{a b}\right)=0, \quad \varepsilon\left(B^{a}\right)=\varepsilon\left(C^{\mu a}\right)=1 .
$$

In accordance with the quantization rules [6], the set of the fields $\phi^{A}$ is supplemented by the corresponding sets of variables $\phi_{A a}^{*}, \pi^{A a}$ and $\bar{\phi}_{A}$,

$$
\begin{aligned}
\phi_{A a}^{*} & =\left(A_{\mu a}^{*}, H_{\mu \nu a}^{*} ; B_{\mu a}^{*}, B_{a \mid b}^{*} ; C_{\mu a \mid b}^{*}, C_{a \mid b c}^{*}\right), \\
\pi^{A a} & =\left(\pi_{(A)}^{\mu a}, \pi_{(H)}^{\mu \nu a} ; \pi_{(B)}^{\mu a}, \pi_{(B)}^{a \mid b} ; \pi_{(C)}^{\mu a \mid b}, \pi_{(C)}^{a \mid b c}\right), \\
\bar{\phi}_{A} & =\left(\bar{A}_{\mu}, \bar{H}_{\mu \nu} ; \bar{B}_{\mu}, \bar{B}_{a} ; \bar{C}_{\mu a}, \bar{C}_{a b}\right),
\end{aligned}
$$

as well as by the auxiliary variables $\lambda^{A}$,

$$
\lambda^{A}=\left(\lambda_{(A)}^{\mu}, \lambda_{(H)}^{\mu \nu} ; \lambda_{(B)}^{\mu}, \lambda_{(B)}^{a} ; \lambda_{(C)}^{\mu a}, \lambda_{(C)}^{a b}\right)
$$

with the following Grassmann parities

$$
\varepsilon\left(\phi_{A a}^{*}\right)=\varepsilon\left(\pi^{A a}\right)=\varepsilon\left(\phi^{A}\right)+1, \quad \varepsilon\left(\bar{\phi}_{A}\right)=\varepsilon\left(\lambda^{A}\right)=\varepsilon\left(\phi^{A}\right) .
$$

\footnotetext{
${ }^{4}$ In the following the group index $p=1, \ldots, N$ will be omitted
} 
The ghost number is assigned to the fields and auxiliary variables by the rule that $a=1$ and $a=2$ bears ghost number +1 and -1 , respectively, for upper indices, as well as -1 and +1 , respectively, for lower indices. The "external" index $a$ on the variables $\phi_{A a}^{*}$ and $\pi^{A a}$ is independent from the (symmetrized) "internal" ones and, therefore, separated by a vertical stroke "|".

An explicit solution of the generating equations (1.1) for the model in question can be found in a closed form as follows:

$$
\begin{aligned}
W=\int d^{4} x( & \left.-\frac{1}{4} \varepsilon^{\mu \nu \rho \sigma} F_{\mu \nu} H_{\rho \sigma}+\frac{1}{2} A_{\mu} A^{\mu}\right) \\
+\int d^{4} x & \left\{H_{\mu \nu a}^{*}\left(\mathcal{D}^{\mu} C^{\nu a}-\mathcal{D}^{\nu} C^{\mu a}\right)-\varepsilon^{a b} C_{\mu a \mid b}^{*} B^{\mu}+\bar{H}_{\mu \nu}\left(\mathcal{D}^{\mu} B^{\nu}-\mathcal{D}^{\nu} B^{\mu}\right)\right. \\
& +C_{\mu a \mid b}^{*} \mathcal{D}^{\mu} C^{a b}-\varepsilon^{a b} C_{a \mid b c}^{*} B^{c}-\frac{1}{2} B_{\mu a}^{*} \mathcal{D}^{\mu} B^{a}+\bar{C}_{\mu a} \mathcal{D}^{\mu} B^{a} \\
& \left.+\frac{1}{2} \varepsilon^{\mu \nu \rho \sigma}\left(H_{\mu \nu a}^{*} \wedge H_{\rho \sigma b}^{*}\right) C^{a b}-\frac{1}{2} \varepsilon^{\mu \nu \rho \sigma}\left(H_{\mu \nu a}^{*} \wedge \bar{H}_{\rho \sigma}\right) B^{a}\right\},
\end{aligned}
$$

where, after having omitted the gauge indices, we use the notations $A^{p} B^{p}=A B, \mathcal{D}_{\mu} B=$ $\partial_{\mu} B+A_{\mu} \wedge B,(A \wedge B)^{p}=f^{p q r} A^{q} B^{r}$. It is easy to see that $\Delta^{a} W \equiv 0$ holds. This action, after appropriate redefinitions of variables, coincides with the corresponding action obtained in [29] (see eq. (31) therein). Note also that the antifields related to $H$ appear bilinear in that action.

A minimal admissible solution of the generating equation (1.2) for the gauge-fixing functional $X$ can be represented as

$$
\begin{aligned}
X=\int d^{4} x & \left\{\bar{A}_{\mu} \lambda_{(A)}^{\mu}+\left(\bar{H}_{\mu \nu}+\frac{\alpha}{2} H_{\mu \nu}\right) \lambda_{(H)}^{\mu \nu}+\bar{B}_{\mu} \lambda_{(B)}^{\mu}+\bar{B}_{a} \lambda_{(B)}^{\mu}+\left(\bar{C}_{\mu a}-\beta \varepsilon_{a b} C_{\mu}^{b}\right) \lambda_{(C)}^{\mu a}\right. \\
& \left.+\bar{C}_{a b} \lambda_{(C)}^{a b}+\frac{\alpha}{4} \pi_{(H) \mu \nu}^{a} \pi_{(H)}^{\mu \nu b}-\frac{\beta}{2} \varepsilon_{a b} \varepsilon_{c d} \pi_{(C) \mu}^{a \mid c} \pi_{(C)}^{\mu b \mid d}\right\},
\end{aligned}
$$

with $\alpha$ and $\beta$ being constant parameters. Here, let us remark that the functional $F$, eq. (1.5), has been chosen as $F\left(H^{\mu \nu}, C^{\mu a}\right)=-\int d^{4} x\left(\frac{1}{4} \alpha H_{\mu \nu} H^{\mu \nu}+\frac{1}{2} \beta \varepsilon_{a b} C_{\mu}^{a} C^{b \mu}\right)$.

Now, substituting the solutions of $W$, eq. (2.5), and $X$, eq. (2.6), into eq. (1.4), we obtain the corresponding vacuum functional $Z$, with the integrand according to eq. (1.6) being invariant under the following symmetry transformations:

$$
\begin{aligned}
\delta A^{\alpha}= & \pi_{(A)}^{\alpha a} \mu_{a} \\
\delta H^{\alpha \beta}= & \pi_{(H)}^{\alpha \beta a} \mu_{a}-\left(\mathcal{D}^{[\alpha} C^{\beta] a}+\varepsilon^{\alpha \beta \gamma \delta} H_{\gamma \delta b}^{*} \wedge C^{a b}-\frac{1}{2} \varepsilon^{\alpha \beta \gamma \delta} \bar{H}_{\gamma \delta} \wedge B^{a}\right) \mu_{a}, \\
\delta B^{\alpha}= & \pi_{(B)}^{\alpha a} \mu_{a}+\frac{1}{2} \mathcal{D}^{\alpha} B^{a} \mu_{a}, \\
\delta B^{a}= & \pi_{(B)}^{b \mid a} \mu_{b}, \\
\delta C^{\alpha a}= & \pi_{(C)}^{\alpha b \mid a} \mu_{b}-\left(\varepsilon^{a b} B^{\alpha}+\mathcal{D}^{\alpha} C^{a b}\right) \mu_{b}, \\
\delta C^{a b}= & \pi_{(C)}^{c \mid a b} \mu_{c}+\frac{1}{2} \varepsilon^{c\{a} B^{b\}} \mu_{c}, \\
\delta A_{\alpha a}^{*}= & \mu_{a}\left(A_{\alpha}-\frac{1}{2} \varepsilon_{\alpha \beta \gamma \delta} \mathcal{D}^{\beta} H^{\gamma \delta}-2 H_{\alpha \beta b}^{*} \wedge C^{\beta b}-2 \bar{H}_{\alpha \beta} \wedge B^{\beta}\right. \\
& \left.-C_{\alpha b \mid c}^{*} \wedge C^{b c}+\left(\frac{1}{2} B_{\alpha b}^{*}-\bar{C}_{\alpha b}\right) \wedge B^{b}\right),
\end{aligned}
$$




$$
\begin{aligned}
& \delta H_{\alpha \beta a}^{*}=-\mu_{a}\left(\frac{1}{4} \varepsilon_{\alpha \beta \gamma \delta} F^{\gamma \delta}+\frac{\alpha}{2} \lambda_{(H) \alpha \beta}\right), \\
& \delta B_{\alpha a}^{*}=\mu_{a}\left(2 \mathcal{D}^{\beta} \bar{H}_{\alpha \beta}-\varepsilon^{b c} C_{\alpha b \mid c}^{*}\right), \\
& \delta B_{a \mid b}^{*}=\mu_{a}\left(\varepsilon^{c d} C_{c \mid b d}^{*}+\frac{1}{2} \mathcal{D}^{\alpha} B_{\alpha b}^{*}-\mathcal{D}^{\alpha} \bar{C}_{\alpha b}-\frac{1}{2} \varepsilon^{\alpha \beta \gamma \delta} H_{\alpha \beta b}^{*} \wedge \bar{H}_{\gamma \delta}\right), \\
& \delta C_{\alpha a \mid b}^{*}=\mu_{a}\left(2 \mathcal{D}^{\beta} H_{\alpha \beta b}^{*}+\beta \varepsilon_{b c} \lambda_{(C) \alpha}^{c}\right), \\
& \delta C_{a \mid b c}^{*}=\mu_{a}\left(-\frac{1}{2} \mathcal{D}^{\alpha} C_{\alpha\{b \mid c\}}^{*}+\frac{1}{2} \varepsilon^{\alpha \beta \gamma \delta} H_{\alpha \beta b}^{*} \wedge H_{\gamma \delta \mid c}^{*}\right), \\
& \delta \pi_{(A)}^{\alpha a}=-\varepsilon^{a b} \lambda_{(A)}^{\alpha} \mu_{b} \\
& \delta \pi_{(H)}^{\alpha \beta a}=-\varepsilon^{a b} \lambda_{(H)}^{\alpha \beta} \mu_{b}+\varepsilon^{a b}\left(\mathcal{D}^{[\alpha} B^{\beta]}+\frac{1}{2} \varepsilon^{\alpha \beta \gamma \delta} H_{\delta \gamma c}^{*} \wedge B^{c}\right) \mu_{b}, \\
& \delta \pi_{(B)}^{\alpha a}=-\varepsilon^{a b} \lambda_{(B)}^{\alpha} \mu_{b} \\
& \delta \pi_{(B)}^{a \mid b}=-\varepsilon^{a c} \lambda_{(B)}^{b} \mu_{c} \\
& \delta \pi_{(C)}^{\alpha a \mid b}=-\varepsilon^{a c} \lambda_{(C)}^{\alpha b} \mu_{c}+\varepsilon^{a c} \mathcal{D}^{\alpha} B^{b} \mu_{c}, \\
& \delta \pi_{(C)}^{a \mid b c}=-\varepsilon^{a d} \lambda_{(C)}^{b c} \mu_{d}, \\
& \delta \bar{A}_{\alpha}=\mu_{a} \varepsilon^{a b} A_{\alpha b}^{*}, \\
& \delta \bar{H}_{\alpha \beta}=\mu_{a}\left(\varepsilon^{a b} H_{\alpha \beta b}^{*}-\frac{\alpha}{2} \pi_{(H) \alpha \beta}^{a}\right) \text {, } \\
& \delta \bar{B}_{\alpha}=\mu_{a} \varepsilon^{a b} B_{\alpha b}^{*}, \\
& \delta \bar{B}_{a}=\mu_{c} \varepsilon^{c b} B_{b \mid a}^{*}, \\
& \delta \bar{C}_{\alpha a}=\mu_{c}\left(\varepsilon^{c b} C_{\alpha b \mid a}^{*}+\beta \varepsilon_{a b} \pi_{(C)^{\alpha}}^{c \mid b}\right), \\
& \delta \bar{C}_{a b}=\mu_{c} \varepsilon^{c d} C_{d \mid a b}^{*},
\end{aligned}
$$

where symmetrization and antisymmetrization is taken as $A^{\{a b\}}=A^{a b}+A^{b a}$ and $A^{[\alpha \beta]}=$ $A^{\alpha \beta}-A^{\beta \alpha}$, respectively. Eqs. (2.7) realize the transformations of extended BRST symmetry of the vacuum functional in terms of the anticanonically conjugated variables $\left(\phi^{A}\right.$, $\left.\phi_{A a}^{*}\right)$ and $\left(\pi^{A a}, \bar{\phi}_{A}\right)$.

Integrating in eq. (1.4) over the variables $\phi_{A a}^{*}, \pi^{A a}, \bar{\phi}_{A}$ and $\lambda^{A}$, we represent the vacuum functional $Z$ as an integral over the fields $\phi^{A}$ of the complete configuration space,

$$
Z=\int d \phi \Delta \exp \left\{\frac{i}{\hbar} S_{\mathrm{eff}}^{(0)}(\phi)\right\}
$$

where

$$
\begin{aligned}
S_{\mathrm{eff}}^{(0)}= & \int d^{4} x\left(-\frac{1}{4} \varepsilon^{\mu \nu \rho \sigma} F_{\mu \nu} H_{\rho \sigma}+\frac{1}{2} A_{\mu} A^{\mu}\right) \\
& +\int d^{4} x\left\{\frac{\alpha}{4} G_{\mu \nu}^{a} \mathcal{M}_{a b}^{-1} \mathcal{K}_{c}^{b[\mu \nu][\rho \sigma]} G_{\rho \sigma}^{c}-\frac{\beta}{2} \varepsilon_{a b} \varepsilon_{c d}\left(\mathcal{D}_{\mu} C^{a c}\right)\left(\mathcal{D}^{\mu} C^{b d}\right)\right\} \\
& +\int d^{4} x\left(\alpha B_{\mu} \mathcal{D}_{\nu} H^{\nu \mu}+\beta\left(\varepsilon_{a b} B^{a} \mathcal{D}_{\mu} C^{\mu b}-B_{\mu} B^{\mu}\right)\right) \\
\Delta= & \int d H^{*} \exp \left\{\frac{2 i}{\alpha \hbar} \int d^{4} x H_{0 i a}^{*} \mathcal{M}^{a b} H_{0 j b}^{*} \eta^{i j}\right\}
\end{aligned}
$$


In eq. (2.9), we have used the following notations:

$$
\begin{aligned}
\mathcal{K}_{b}^{a[\mu \nu][\rho \sigma]} & \equiv \frac{1}{2}\left\{\delta_{b}^{a}\left(\eta^{\mu \rho} \eta^{\nu \sigma}-\eta^{\mu \sigma} \eta^{\nu \rho}\right)+\alpha \mathcal{C}_{b}^{a} \varepsilon^{\mu \nu \rho \sigma}\right\} \\
G_{\mu \nu}^{a} & \equiv\left(\mathcal{D}_{\mu} C_{\nu}^{a}-\mathcal{D}_{\nu} C_{\mu}^{a}\right)-\frac{\alpha}{4} \varepsilon_{\mu \nu \rho \sigma} \mathcal{B}^{a} H^{\rho \sigma}
\end{aligned}
$$

The matrix $\mathcal{M}_{a b}^{-1}$ in $(2.10)$ is the inverse of $\mathcal{M}^{a b}$,

$$
\mathcal{M}^{a b} \equiv \varepsilon^{a b}-\alpha^{2} \mathcal{C}_{c}^{a} \mathcal{C}_{d}^{b} \varepsilon^{c d}, \quad \mathcal{M}^{a c} \mathcal{M}_{c b}^{-1}=\delta_{b}^{a}
$$

here the matrices $\mathcal{C}_{b}^{a}$ and $\mathcal{B}^{a}$ are defined by

$$
\mathcal{C}_{b}^{a} E \equiv \varepsilon_{b c} C^{a c} \wedge E, \quad \mathcal{B}^{a} E \equiv B^{a} \wedge E
$$

The functional $S_{\text {eff }}^{(0)}$ in eq. (2.9) is the tree approximation to the gauge-fixed quantum action of the theory, while the functional $\Delta$ in eq. (2.10) can be considered as a contribution to the integration measure. (The proof of the above representation of the vacuum functional (2.8) is given in the Appendix).

The integrand in eq. (2.8) is invariant under the following symmetry transformations:

$$
\begin{aligned}
\delta A_{\mu} & =0 \\
\delta H^{\alpha \beta} & =-\varepsilon^{a b} \mathcal{M}_{b c}^{-1} \mathcal{K}_{d}^{c[\alpha \beta][\gamma \delta]} G_{\gamma \delta}^{d} \mu_{a} \\
\delta B^{\alpha} & =\frac{1}{2} \mathcal{D}^{\alpha} B^{a} \mu_{a} \\
\delta B^{a} & =0 \\
\delta C^{\alpha a} & =\left(\mathcal{D}^{\alpha} C^{a b}-\varepsilon^{a b} B^{\alpha}\right) \mu_{b} \\
\delta C^{a b} & =\frac{1}{2} B^{\{a} \varepsilon^{b\} c} \mu_{c} .
\end{aligned}
$$

These transformations are the (anti)BRST transformations of the $S p(2)$ invariant action $S_{\text {eff }}^{(0)}$ which, together with the integration measure $d \phi \Delta$ in eq. (2.8), is left invariant:

$$
\begin{gathered}
\delta(d \phi)=d \phi \delta^{4}(0) \int d^{4} x \operatorname{Tr} \mathcal{W}, \\
\delta \Delta=-\Delta \delta^{4}(0) \int d^{4} x \operatorname{Tr} \mathcal{W}, \\
\delta\left(\exp \left\{\frac{i}{\hbar} S_{\text {eff }}^{(0)}\right\}\right)=0
\end{gathered}
$$

where the following notations have been used:

$$
\mathcal{W}=-3 \alpha^{2} \varepsilon^{a b}\left(\mathcal{M}_{b c}^{-1} \mathcal{C}_{d}^{c} \mathcal{B}^{d}\right) \mu_{a}, \quad \operatorname{Tr} \mathcal{W} \equiv \sum_{p=1}^{N} \mathcal{W}^{p p}
$$

Consequently, eqs. (2.14) realize the transformations of extended BRST symmetry for the vacuum functional (2.8) in terms of the variables $\phi^{A}$ of the complete configuration space. Remarkably, the (anti)BRST transformations of the classical field $H^{\alpha \beta}$ essentially depend on the gauge parameter $\alpha$ whereas all the others coincide - up to the $\pi$-terms with the transformations (2.7). T The appearance of the parameter $\alpha$ can be traced back to the non-linear dependence of the extended action $S$ on the antifields $H_{\mu \nu a}^{*}$.

\footnotetext{
${ }^{5}$ To observe this fact, it is sufficient to make the replacements $\mu_{a} \rightarrow-\mu_{a}$ and $B^{\mu} \rightarrow-B^{\mu}$.
} 
Note that, taking into account the action (2.9) and the contribution to the integration measure (2.10), the vacuum functional (2.8) obtained for the Freedman-Townsend model leads to the unitarity [25] of the physical $S$ matrix (for discussions of the unitarity problem in the case of this model, see also [24, 26, 27]).

For the first time, the covariant quantization of the Freedman-Townsend model in the framework of extended BRST invariance has been performed by Barnich, Constantinescu and Gregoire [29]. However, these authors used a more complicated, $S p(2)$-noncovariant method of gauge fixing which might have prevented them from explicitly solving their expression with respect to the fields $\phi^{A}$ completely. Namely, they left the dependence of the action from the antifields $H_{\alpha \beta}^{*}$ and $\bar{H}_{\alpha \beta}$ which we were able to integrate out. Similar results have been obtained in Ref. [30], where it has been shown that the elimination of antifields leads to a nonlocal action in terms of the fields. The same conclusion could be drawn from our result since the matrix $\mathcal{M}^{-1}$ is given as an infinite series only.

\section{3. $W_{2}$-gravity}

The model of $W_{2}$-gravity [18] is described by the action

$$
S_{c l}(\varphi, h)=\frac{1}{2 \pi} \int d^{2} z\left(\partial \varphi \bar{\partial} \varphi-h(\partial \varphi)^{2}\right)
$$

where $\varphi$ and $h$ are bosonic classical fields, $\varepsilon(\varphi)=\varepsilon(h)=0$, defined on a space with complex coordinates, $(z, \bar{z})$, so that $\partial=\partial / \partial z, \bar{\partial}=\partial / \partial \bar{z}$.

The action (3.1) is invariant under the gauge transformations

$$
\begin{aligned}
\delta \varphi & =(\partial \varphi) \xi \\
\delta h & =\bar{\partial} \xi-h \partial \xi+(\partial h) \xi
\end{aligned}
$$

with the gauge function $\xi(z, \bar{z})$. These transformations form a closed algebra,

$$
\begin{aligned}
& {\left[\delta_{\xi(1)}, \delta_{\xi(2)}\right]=\delta_{\xi(1,2)}} \\
& \xi_{(1,2)}=\left(\partial \xi_{(1)}\right) \xi_{(2)}-\left(\partial \xi_{(2)}\right) \xi_{(1)} .
\end{aligned}
$$

Note that the quantum properties of $W_{2}$-gravity, considered within the BV method [16], have been discussed in [21, 31, 32], where also the one-loop anomaly has been calculated. Recently, the quantization of that model has been performed in the triplectic formalism [33], however, with another kind of gauge fixing being different from using the gauge fixing functional $X$.

Now, we consider the gauge model (3.1), (3.2) and (3.3) in the framework of the modified triplectic quantization. First, let us introduce the complete configuration space

$\phi^{A}$, whose structure in the case of the model in question is determined by the rules of the $S p(2)$ formalism for irreducible gauge theories. Thus, the space of the variables $\phi^{A}$ is constructed by supplementing the initial space of the fields $(\varphi, h)$ with the doublet $C^{a}, \varepsilon\left(C^{a}\right)=1$, of Faddeev-Popov ghosts, and the Lagrange multiplier $B, \varepsilon(B)=0$, corresponding to the gauge parameter $\xi$ in eq. (3.2).

The fields $\phi^{A}$ of the complete configuration space,

$$
\phi^{A}=\left(\varphi, h ; B, C^{a}\right),
$$


are supplemented by the sets of the variables $\phi_{A a}^{*}, \pi^{A a}$ and $\bar{\phi}_{A}$,

$$
\begin{aligned}
\phi_{A a}^{*} & =\left(\varphi_{a}^{*}, h_{a}^{*} ; B_{a}^{*}, C_{a \mid b}^{*}\right), \\
\pi^{A a} & =\left(\pi_{(\varphi)}^{a}, \pi_{(h)}^{a} ; \pi_{(B)}^{a}, \pi_{(C)}^{a \mid b}\right), \\
\bar{\phi}_{A} & =\left(\bar{\varphi}, \bar{h} ; \bar{B}, \bar{C}_{a}\right),
\end{aligned}
$$

as well as by the additional variables $\lambda^{A}$,

$$
\lambda^{A}=\left(\lambda_{(\varphi)}, \lambda_{(h)} ; \lambda_{(B)}, \lambda_{(C)}^{a}\right),
$$

with the Grassmann parities given by eq. (2.4).

An action functional $S$ of the gauge model (3.1), (3.2) and (3.3) satisfying the generating equations (1.1) in tree approximation, i.e., when ignoring their r.h.s., is given as follows:

$$
\begin{aligned}
S= & \frac{1}{2 \pi} \int d^{2} z\left(\partial \varphi \bar{\partial} \varphi-h(\partial \varphi)^{2}\right) \\
& +\int d^{2} z\left\{\varphi_{a}^{*} C^{a} \partial \varphi+h_{a}^{*}\left(\bar{\partial} C^{a}-h \partial C^{a}+C^{a} \partial h\right)\right. \\
& +\left(\frac{1}{2} B_{a}^{*}-\bar{C}_{a}\right)\left[\left(C^{a} \partial B-B \partial C^{a}\right)+\frac{1}{6} \varepsilon_{b d}\left(C^{\{a}\left(\partial^{2} C^{d\}}\right) C^{b}-C^{\{a}\left(\partial C^{d\}}\right) \partial C^{b}\right)\right] \\
& -C_{a \mid b}^{*}\left(\varepsilon^{a b} B+\frac{1}{2} C^{\{a} \partial C^{b\}}\right)+\bar{\varphi}\left(B \partial \varphi+\frac{1}{2} \varepsilon_{a b} C^{a} \partial\left(C^{b} \partial \varphi\right)\right)+\bar{h}(\bar{\partial} B-h \partial B+B \partial h) \\
& \left.+\frac{1}{2} \varepsilon_{a b} \bar{h}\left(C^{a} \partial\left(\bar{\partial} C^{b}-h \partial C^{b}+C^{b} \partial h\right)+\left(\bar{\partial} C^{b}-h \partial C^{b}+C^{b} \partial h\right) \partial C^{a}\right)\right\} .
\end{aligned}
$$

Obviously, the application of the differential operator $\Delta^{a}$ leads to terms being proportional to $\delta(0)$. However, since the model is strictly two-dimensional the dimensional regularization is not applicable. Therefore, it is not surprising that the model has an anomaly which can not be compensated by appropriate counterterms.

Furthermore, a solution of the generating equations determining the gauge-fixing functional $X$ can be represented as

$$
\begin{gathered}
X=\int d^{2} z\left\{(\bar{\varphi}-\alpha \varphi-\beta h) \lambda_{(\varphi)}+(\bar{h}-\beta \varphi-\gamma h) \lambda_{(h)}+\bar{B} \lambda_{(B)}+\bar{C}_{a} \lambda_{(C)}^{a}\right. \\
\left.-\frac{\alpha}{2} \varepsilon_{a b} \pi_{(\varphi)}^{a} \pi_{(\varphi)}^{b}-\beta \varepsilon_{a b} \pi_{(\varphi)}^{a} \pi_{(h)}^{b}-\frac{\gamma}{2} \varepsilon_{a b} \pi_{(h)}^{a} \pi_{(h)}^{b}\right\}
\end{gathered}
$$

with $\alpha, \beta$ and $\gamma$ being constant parameters. Here, $F$ has been chosen as $F(\varphi, h)=$ $\int d^{2} z\left(\frac{1}{2} \alpha \varphi^{2}+\beta \varphi h+\frac{1}{2} \gamma h^{2}\right)$.

The vacuum functional (1.4) corresponding to the solutions (3.4) and (3.5) of the generating equations that determine the action $W$ in tree approximation and the gaugefixing functional $X$, is invariant under the following transformations of extended BRST symmetry, expressed (for simplicity) in terms of the derivatives of $S$ :

$$
\begin{aligned}
\delta \phi^{A} & =\left(\pi^{A a}-\frac{\delta S}{\delta \phi_{A a}^{*}}\right) \mu_{a} \\
\delta \varphi_{a}^{*} & =\mu_{a}\left(\frac{\delta S}{\delta \varphi}+\alpha \lambda_{(\varphi)}+\beta \lambda_{(h)}\right), \\
\delta h_{a}^{*} & =\mu_{a}\left(\frac{\delta S}{\delta h}+\beta \lambda_{(\varphi)}+\gamma \lambda_{(h)}\right),
\end{aligned}
$$




$$
\begin{aligned}
\delta B_{a}^{*} & =\mu_{a} \frac{\delta S}{\delta B}, \\
\delta C_{a \mid b}^{*} & =\mu_{a} \frac{\delta S}{\delta C^{b}}, \\
\delta \pi^{A a} & =\varepsilon^{a b}\left(\frac{\delta S}{\delta \bar{\phi}^{A}}-\lambda^{A}\right) \mu_{b}, \\
\delta \bar{\varphi} & =\mu_{a}\left(\varepsilon^{a b} \varphi_{b}^{*}+\alpha \pi_{(\varphi)}^{a}+\beta \pi_{(h)}^{a}\right), \\
\delta \bar{h} & =\mu_{a}\left(\varepsilon^{a b} h_{b}^{*}+\beta \pi_{(\varphi)}^{a}+\gamma \pi_{(h)}^{a}\right), \\
\delta \bar{B} & =\mu_{a} \varepsilon^{a b} B_{b}^{*}, \\
\delta \overline{C_{a}} & =\mu_{b} \varepsilon^{b d} C_{d \mid a}^{*} .
\end{aligned}
$$

Substituting the solutions (3.4), (3.5) for the action $S$ and the gauge-fixing functional $X$ into eq. (1.4), and integrating out the variables $\phi_{A a}^{*}, \pi^{A a}, \bar{\phi}_{A}, \lambda^{A}$, we obtain the vacuum functional $Z$ as an integral over the fields $\phi^{A}$ of the complete configuration space,

$$
Z=\int d \phi \exp \left\{\frac{i}{\hbar} S_{\text {eff }}(\phi)\right\},
$$

where $S_{\text {eff }}$ is the gauge-fixed tree approximation of the quantum action

$$
\begin{aligned}
S_{\mathrm{eff}}= & \frac{1}{2 \pi} \int d^{2} z\left(\partial \varphi \bar{\partial} \varphi-h(\partial \varphi)^{2}\right) \\
& +\int d^{2} z[(\alpha \varphi+\beta h) B \partial \varphi+(\beta \varphi+\gamma h)(\bar{\partial} B-h \partial B+B \partial h)] \\
& +\frac{1}{2} \varepsilon_{a b} \int d^{2} z\left[\left(\alpha C^{b} \partial \varphi+\beta\left(\bar{\partial} C^{b}-h \partial C^{b}+C^{b} \partial h\right)\right) C^{a} \partial \varphi-(\alpha \varphi+\beta h) C^{a} \partial\left(C^{b} \partial \varphi\right)\right. \\
& +\left(\beta C^{b} \partial \varphi+\gamma\left(\bar{\partial} C^{b}-h \partial C^{b}+C^{b} \partial h\right)\right)\left(\bar{\partial} C^{a}-h \partial C^{a}+C^{a} \partial h\right) \\
& \left.-(\beta \varphi+\gamma h)\left(C^{a} \partial\left(\bar{\partial} C^{b}-h \partial C^{b}+C^{b} \partial h\right)+\left(\bar{\partial} C^{b}-h \partial C^{b}+C^{b} \partial h\right) \partial C^{a}\right)\right] . \quad(3.8)
\end{aligned}
$$

The quantum action $S_{\text {eff }}$, eq. (3.8), and the integration measure are invariant under the following (anti)BRST transformations:

$$
\begin{aligned}
\delta \varphi & =C^{a} \partial \varphi \mu_{a}, \\
\delta h & =\left(\bar{\partial} C^{a}-h \partial C^{a}+C^{a} \partial h\right) \mu_{a}, \\
\delta B & =\frac{1}{2}\left(C^{a} \partial B-B \partial C^{a}\right) \mu_{a}+\frac{1}{12} \varepsilon_{b d}\left(C^{\{a}\left(\partial^{2} C^{d\}}\right) C^{b}-C^{\{a}\left(\partial C^{d\}}\right) \partial C^{b}\right) \mu_{a}, \\
\delta C^{a} & =\left(\varepsilon^{a b} B-\frac{1}{2} C^{\{a} \partial C^{b\}}\right) \mu_{b} .
\end{aligned}
$$

Thus we conclude that eqs. (3.9) realize the transformations of extended BRST symmetry in terms of the variables of the complete configuration space. Furthermore, introducing the action of the (anti)BRST operators $s^{a}$ onto the fields $\phi^{A}$ according to the rule $\delta \phi^{A}=\left(s^{a} \phi^{A}\right) \mu_{a}$, one can rewrite the effective action (3.8) in the following compact form:

$$
S_{\text {eff }}=S_{\mathrm{cl}}+\frac{1}{2} \varepsilon_{a b} s^{b} s^{a} F(\varphi, h),
$$

i.e., one obtains the usual effective action of the $S p(2)$-covariant approach. The invariance of $S_{\text {eff }}$ under the transformations (3.9) follows by virtue of the (generalized) nilpotency, $s^{\{a} s^{b\}}=0$. 


\section{Two-dimensional Gravity with Dynamical Torsion}

The theory of two-dimensional gravity with dynamical torsion is described in terms of the zweibein and Lorentz connection $\left(e_{\mu}^{i}, \omega_{\mu}^{i j}=\varepsilon^{i j} \omega_{\mu}\right)$ by the action 19

$$
S_{c l}\left(e_{\mu}^{i}, \omega_{\mu}\right)=\int d^{2} x e\left(\frac{1}{16 \alpha} R_{\mu \nu}^{i j} R^{\mu \nu}{ }_{i j}-\frac{1}{8 \beta} T_{\mu \nu}{ }^{i} T_{i}^{\mu \nu}-\gamma\right)
$$

where $\alpha, \beta$ and $\gamma$ are constant parameters. In eq. (4.1), the Latin indices are lowered with the help of the Minkowski metric $\eta_{i j}=\operatorname{diag}(+1,-1)$, and the Greek indices, with the help of the metric tensor $g_{\mu \nu}=\eta_{i j} e_{\mu}^{i} e_{\nu}^{j}$. Besides, the following notations are used:

$$
\begin{aligned}
e & =\operatorname{det} e_{\mu}^{i}, \\
R_{\mu \nu}^{i j} & =\varepsilon^{i j} \partial_{\mu} \omega_{\nu}-(\mu \leftrightarrow \nu), \\
T_{\mu \nu}^{i} & =\partial_{\mu} e_{\nu}^{i}+\varepsilon^{i j} \omega_{\mu} e_{\nu j}-(\mu \leftrightarrow \nu),
\end{aligned}
$$

where $\epsilon^{i j}$ is a constant antisymmetric tensor, $\epsilon^{01}=-1$.

Note that the model (4.1) is the most general theory of two-dimensional $R^{2}$-gravity with independent dynamical torsion that leads to second-order equations of motion for the zweibein and Lorentz connection. Thus, supplementing the action eq. (4.1) by the Einstein-Hilbert term $e R$ would not affect the classical field equations, since in two dimensions it reduces to a trivial total divergence.

Originally, the action (4.1) was proposed [34] in the context of bosonic string theory, where it was used to describe the dynamics of string geometry. There, moreover, it was proved that the string with dynamical geometry has no critical dimension.

An attractive feature of the model (4.1) is its complete integrability. The corresponding equations of motion have been studied in conformal [19, 35] and light-cone [36] gauges. 9 It was established that the model contains solutions with constant curvature and zero torsion, thus incorporating several other two-dimensional gravity models 338 whose actions, however, do not have a purely geometric interpretation.

The action (4.1) is invariant under local Lorentz rotations of the zweibein $e_{\mu}^{i}$, which infinitesimally imples the gauge transformations

$$
\delta_{\zeta} e_{\mu}^{i}=\varepsilon^{i j} e_{\mu j} \zeta, \quad \delta_{\zeta} \omega_{\mu}=-\partial_{\mu} \zeta
$$

with the parameter $\zeta$. Similarly, the general coordinate invariance of eq. (4.1) leads to the gauge transformations

$$
\delta_{\xi} e_{\mu}^{i}=e_{\nu}^{i} \partial_{\mu} \xi^{\nu}+\left(\partial_{\nu} e_{\mu}^{i}\right) \xi^{\nu}, \quad \delta_{\xi} \omega_{\mu}=\omega_{\nu} \partial_{\mu} \xi^{\nu}+\left(\partial_{\nu} \omega_{\mu}\right) \xi^{\nu}
$$

with the parameters $\xi^{\mu}$. The gauge transformations (4.2) and (4.3) form a closed algebra

$$
\begin{aligned}
{\left[\delta_{\zeta(1)}, \delta_{\zeta(2)}\right] } & =0, \\
{\left[\delta_{\xi(1)}, \delta_{\xi(2)}\right] } & =\delta_{\xi(1,2)}, \\
{\left[\delta_{\zeta}, \delta_{\xi}\right] } & =\delta_{\zeta^{\prime}},
\end{aligned}
$$

where

$$
\xi_{(1,2)}^{\mu}=\left(\partial_{\nu} \xi^{\mu}{ }_{(1)}\right) \xi_{(2)}^{\nu}-\left(\partial_{\nu} \xi^{\mu}{ }_{(2)}\right) \xi_{(1)}^{\nu}, \quad \zeta^{\prime}=\left(\partial_{\mu} \zeta\right) \xi^{\mu}
$$

\footnotetext{
${ }^{6} \mathrm{~A}$ convenient general framework for the study of this and other two-dimensional theories is also provided by the Poisson- $\sigma$ model approach [37].
} 
Note that in Ref. [39] a gauge model classically equivalent to (4.1), (4.2), (4.3) and (4.4) was proposed by means of artificially adding the Einstein-Hilbert term coupled to an additional scalar field, $\sigma e R$; however, in this equivalent formulation the algebra of the corresponding gauge transformations closes only on-shell.

The Hamiltonian structure of the gauge symmetries of the original model was studied in Ref. [40, and its canonical quantization, in Ref. [41]. Quantum properties of the model in the light-cone gauge were discussed in Ref. [42], proving also, despite of the nonpolynomial structure of the theory, its renormalizability. Its quantization within the $S p(2)$ covariant approach has been considered in Refs. 43.

Now, we consider the gauge model (4.1), (4.2), (4.3) and (4.4) in the framework of the modified triplectic formalism [6].

The complete configuration space $\phi^{A}$, constructed by the rules of the $S p(2)$ covariant quantization of irreducible theories, consists of the initial classical fields $\left(e_{\mu}^{i}, \omega_{\mu}\right)$, the doublets of the Faddeev-Popov ghosts $\left(C^{a}, C^{\mu a}\right)$ and the Lagrangian multipliers $\left(B, B^{\mu}\right)$ introduced according to the number of the gauge parameters in eqs. (4.2) and (4.3), i.e., $\zeta$ and $\xi^{\mu}$, respectively. The Grassmann parities of the fields $\phi^{A}$,

$$
\phi^{A}=\left(e_{\mu}^{i}, \omega_{\mu} ; B, B^{\mu} ; C^{a}, C^{\mu a}\right)
$$

are given by

$$
\varepsilon\left(e_{\mu}^{i}\right)=\varepsilon\left(\omega_{\mu}\right)=\varepsilon(B)=\varepsilon\left(B^{\mu}\right)=0, \quad \varepsilon\left(C^{a}\right)=\varepsilon\left(C^{\mu a}\right)=1 .
$$

The fields $\phi^{A}$ of the complete configuration space are supplemented by the following sets of the variables $\phi_{A a}^{*}, \pi^{A a}, \bar{\phi}_{A}$ and $\lambda^{A}$ :

$$
\begin{aligned}
\phi_{A a}^{*} & =\left(e_{i a}^{* \mu}, \omega_{a}^{* \mu} ; B_{a}^{*}, B_{\mu a}^{*} ; C_{a \mid b}^{*}, C_{\mu a \mid b}^{*}\right), \\
\pi^{A a} & =\left(\pi_{(e) \mu}^{i a}, \pi_{(\omega) \mu}^{a} ; \pi_{(B)}^{a}, \pi_{(B)}^{\mu a} ; \pi_{(C)}^{a \mid b}, \pi_{(C)}^{\mu a \mid b}\right), \\
\bar{\phi}_{A} & =\left(\bar{e}_{i}^{\mu}, \bar{\omega}^{\mu} ; \bar{B}, \bar{B}_{\mu} ; \bar{C}_{a}, \bar{C}_{\mu a}\right), \\
\lambda^{A} & =\left(\lambda_{(e) \mu}^{i}, \lambda_{(\omega) \mu} ; \lambda_{(B)}, \lambda_{(B)}^{\mu} ; \lambda_{(C)}^{a}, \lambda_{(C)}^{\mu a}\right) .
\end{aligned}
$$

Again, we are faced with the problem that the model is stricly two-dimensional and, therefore, the regularization by setting $\delta(0)=0$ will not be applicable. A functional that satisfies the generating equations (1.1) for the classical action $S$ in this case of the gauge model (4.1), (4.2), (4.3) and (4.4) can be found in a closed form as follows:

$$
\begin{aligned}
S & =\int d^{2} x e\left(\frac{1}{16 \alpha} R_{\mu \nu}{ }^{i j} R^{\mu \nu}{ }_{i j}-\frac{1}{8 \beta} T_{\mu \nu}{ }^{i} T^{\mu \nu}{ }_{i}-\gamma\right) \\
& +\int d^{2} x\left\{e_{i a}^{* \mu}\left(\varepsilon^{i j} e_{\mu j} C^{a}+C^{\lambda a} \partial_{\lambda} e_{\mu}^{i}+e_{\lambda}^{i} \partial_{\mu} C^{\lambda a}\right)+\omega_{a}^{* \mu}\left(-\partial_{\mu} C^{a}+C^{\lambda a} \partial_{\lambda} \omega_{\mu}+\omega_{\lambda} \partial_{\mu} C^{\lambda a}\right)\right. \\
& +\left(\frac{1}{2} B_{a}^{*}-\bar{C}_{a}\right)\left[C^{\mu a} \partial_{\mu} B-B^{\mu} \partial_{\mu} C^{a}+\frac{1}{6} \varepsilon_{b d}\left(C^{\lambda b} \partial_{\lambda}\left(C^{\mu\{a} \partial_{\mu} C^{d\}}\right)-\left(C^{\mu\{a} \partial_{\mu} C^{\lambda d\}}\right) \partial_{\lambda} C^{b}\right)\right] \\
& +\left(\frac{1}{2} B_{\mu a}^{*}-\bar{C}_{\mu a}\right)\left[C^{\lambda a} \partial_{\lambda} B^{\mu}-B^{\lambda} \partial_{\lambda} C^{\mu a}+\frac{1}{6} \varepsilon_{b d}\left[C^{\sigma b} \partial_{\sigma}\left(C^{\lambda\{a} \partial_{\lambda} C^{\mu d\}}\right)-\left(C^{\lambda\{a} \partial_{\lambda} C^{\sigma d\}}\right) \partial_{\sigma} C^{\mu b}\right]\right] \\
& -C_{a \mid b}^{*}\left(\varepsilon^{a b} B+\frac{1}{2} C^{\mu\{a} \partial_{\mu} C^{b\}}\right)-C_{\mu a \mid b}^{*}\left(\varepsilon^{a b} B^{\mu}+\frac{1}{2} C^{\lambda\{a} \partial_{\lambda} C^{\mu b\}}\right) \\
& +\bar{e}_{i}^{\mu}\left[\varepsilon^{i j} B e_{\mu j}+B^{\lambda} \partial_{\lambda} e_{\mu}^{i}+e_{\lambda}^{i} \partial_{\mu} B^{\lambda}+\frac{1}{2} \varepsilon_{a b}\left(\left(e_{\mu}^{i} C^{b}+\varepsilon^{i j} C^{\lambda b} \partial_{\lambda} e_{\mu j}+\varepsilon^{i j} e_{\lambda j} \partial_{\mu} C^{\lambda b}\right) C^{a}\right.\right. \\
& \left.\left.-C^{\lambda a} \partial_{\lambda}\left(\varepsilon^{i j} e_{\mu j} C^{b}+C^{\sigma b} \partial_{\sigma} e_{\mu}^{i}+e_{\sigma}^{i} \partial_{\mu} C^{\sigma b}\right)+\left(\varepsilon^{i j} e_{\lambda j} C^{b}+\left(\partial_{\sigma} e_{\lambda}^{i}\right) C^{\sigma b}+e_{\sigma}^{i} \partial_{\lambda} C^{\sigma b}\right) \partial_{\mu} C^{\lambda a}\right)\right] \\
& +\bar{\omega}^{\mu}\left[-\partial_{\mu} B+B^{\lambda} \partial_{\lambda} \omega_{\mu}+\omega_{\lambda} \partial_{\mu} B^{\lambda}-\frac{1}{2} \varepsilon_{a b}\left(C^{\lambda a} \partial_{\lambda}\left(C^{\sigma b} \partial_{\sigma} \omega_{\mu}+\omega_{\sigma} \partial_{\mu} C^{\sigma b}-\partial_{\mu} C^{b}\right)\right.\right. \\
& \left.\left.\left.-\left(C^{\sigma b} \partial_{\sigma} \omega_{\lambda}+\omega_{\sigma} \partial_{\lambda} C^{\sigma b}-\partial_{\lambda} C^{b}\right) \partial_{\mu} C^{\lambda a}\right)\right]\right\} .
\end{aligned}
$$


A solution of the generating equations determining the gauge-fixing functional $X$ can be chosen as

$$
\begin{aligned}
X=\int d^{2} x & \left\{\left(\bar{e}_{i}^{\mu}-p \eta^{\mu \nu} \eta_{i j} e_{\nu}^{j}\right) \lambda_{(e) \mu}^{i}+\left(\bar{\omega}^{\mu}-q \eta^{\mu \nu} \omega_{\nu}\right) \lambda_{(\omega) \mu}+\bar{B} \lambda_{(B)}+\bar{B}_{\mu} \lambda_{(B)}^{\mu}\right. \\
+ & \left.\bar{C}_{a} \lambda_{(C)}^{a}+\bar{C}_{\mu a} \lambda_{(C)}^{\mu a}-\frac{p}{2} \varepsilon_{a b} \eta_{i j} \eta^{\mu \nu} \pi_{(e) \mu}^{i a} \pi_{(e) \nu}^{j b}-\frac{q}{2} \varepsilon_{a b} \eta^{\mu \nu} \pi_{(\omega) \mu}^{a} \pi_{(\omega) \nu}^{b}\right\},
\end{aligned}
$$

where $F\left(e_{\mu}^{i}, \omega_{\mu}\right)=\frac{1}{2} \int d^{2} x\left(p \eta^{\mu \nu} \eta_{i j} e_{\mu}^{i} e_{\nu}^{j}+q \eta^{\mu \nu} \omega_{\mu} \omega_{\nu}\right)$ has been used with $p, q$ being constant parameters, and $\eta^{\mu \nu}=\operatorname{diag}(+1,-1)$ is the metric of the two-dimensional Minkowski space.

Again, the vacuum functional $Z$ is obtained by substituting the explicit solutions for the tree action $S$, eq. (4.5), and the gauge-fixing functional $X$, eq. (4.6), into the expression eq. (1.4). The symmetry transformations (1.6) will not be given explicitly; their determination is straightforward but the result is quite lenghty.

Performing the integration over the variables $\phi_{A a}^{*}, \pi^{A a}, \bar{\phi}_{A}$ and $\lambda^{A}$, we obtain $Z$ in the form (3.7) with the gauge-fixed effective action $S_{\text {eff }}$,

$$
\begin{aligned}
S_{\text {eff }} & =\int d^{2} x e\left(\frac{1}{16 \alpha} R_{\mu \nu}{ }^{i j} R^{\mu \nu}{ }_{i j}-\frac{1}{8 \beta} T_{\mu \nu}{ }^{i} T^{\mu \nu}{ }_{i}-\gamma\right) \\
& +\int d^{2} x\left\{p \eta^{\mu \nu} e_{\mu i}\left(\varepsilon^{i j} e_{\nu j} B+e_{\lambda}^{i} \partial_{\nu} B^{\lambda}+B^{\lambda} \partial_{\lambda} e_{\nu}^{i}\right)+q \eta^{\mu \nu} \omega_{\mu}\left(\omega_{\lambda} \partial_{\nu} B^{\lambda}+B^{\lambda} \partial_{\lambda} \omega_{\nu}-\partial_{\nu} B\right)\right\} \\
& +\frac{1}{2} \varepsilon_{a b}\left\{p \eta_{i j} \eta^{\mu \nu}\left(\varepsilon^{i k} e_{\nu k} C^{b}+e_{\lambda}^{i} \partial_{\nu} C^{\lambda b}+C^{\lambda b} \partial_{\lambda} e_{\nu}^{i}\right)\left(\varepsilon^{j l} e_{\mu l} C^{a}+e_{\sigma}^{j} \partial_{\mu} C^{\sigma a}+C^{\sigma a} \partial_{\sigma} e_{\mu}^{j}\right)\right. \\
& +p \eta^{\mu \nu} \eta_{i j} e_{\nu}^{j}\left[\left(e_{\mu}^{i} C^{b}+\varepsilon^{i k} e_{\lambda k} \partial_{\mu} C^{\lambda b}+\varepsilon^{i k} C^{\lambda b} \partial_{\lambda} e_{\mu k}\right) C^{a}\right. \\
& \left.+\left(\varepsilon^{i k} e_{\lambda k} C^{b}+e_{\sigma}^{i} \partial_{\lambda} C^{\sigma b}+C^{\sigma b} \partial_{\sigma} e_{\lambda}^{i}\right) \partial_{\mu} C^{\lambda a}-C^{\lambda a} \partial_{\lambda}\left(\varepsilon^{i k} e_{\mu k} C^{b}+e_{\sigma}^{i} \partial_{\mu} C^{\sigma b}+C^{\sigma b} \partial_{\sigma} e_{\mu}^{i}\right)\right] \\
& +q \eta^{\mu \nu}\left(\omega_{\lambda} \partial_{\nu} C^{\lambda b}+C^{\lambda b} \partial_{\lambda} \omega_{\nu}-\partial_{\nu} C^{b}\right)\left(\omega_{\sigma} \partial_{\mu} C^{\sigma a}+C^{\sigma a} \partial_{\sigma} \omega_{\mu}-\partial_{\mu} C^{a}\right) \\
& \left.-q \eta^{\mu \nu} \omega_{\nu}\left[C^{\lambda a} \partial_{\lambda}\left(\omega_{\sigma} \partial_{\mu} C^{\sigma b}+C^{\sigma b} \partial_{\sigma} \omega_{\mu}-\partial_{\mu} C^{b}\right)-\left(\omega_{\sigma} \partial_{\lambda} C^{\sigma b}+C^{\sigma b} \partial_{\sigma} \omega_{\lambda}-\partial_{\lambda} C^{b}\right) \partial_{\mu} C^{\lambda a}\right]\right\} .
\end{aligned}
$$

The effective action $S_{\text {eff }}$ is invariant under the following transformations:

$$
\begin{aligned}
\delta e_{\sigma}^{i} & =\left(\varepsilon^{i j} e_{\sigma j} C^{a}+C^{\lambda a} \partial_{\lambda} e_{\sigma}^{i}+e_{\lambda}^{i} \partial_{\sigma} C^{\lambda a}\right) \mu_{a}, \\
\delta \omega_{\sigma} & =\left(-\partial_{\sigma} C^{a}+C^{\lambda a} \partial_{\lambda} \omega_{\sigma}+\omega_{\lambda} \partial_{\sigma} C^{\lambda a}\right) \mu_{a}, \\
\delta B & =\frac{1}{2}\left(C^{\sigma a} \partial_{\sigma} B-B^{\sigma} \partial_{\sigma} C^{a}+\frac{1}{6} \varepsilon_{b d}\left(C^{\sigma b} \partial_{\sigma}\left(C^{\lambda\{a} \partial_{\lambda} C^{d\}}\right)-\left(C^{\lambda\{a} \partial_{\lambda} C^{\sigma d\}}\right) \partial_{\sigma} C^{b}\right) \mu_{a},\right. \\
\delta B^{\sigma} & =\frac{1}{2}\left(C^{\lambda a} \partial_{\lambda} B^{\sigma}-B^{\lambda} \partial_{\lambda} C^{\sigma a}+\frac{1}{6} \varepsilon_{b d}\left(C^{\lambda b} \partial_{\lambda}\left(C^{\rho\{a} \partial_{\rho} C^{\sigma d\}}\right)-\left(C^{\rho\{a} \partial_{\rho} C^{\lambda d\}}\right) \partial_{\lambda} C^{\sigma b}\right) \mu_{a},\right. \\
\delta C^{a} & =\left(\varepsilon^{a b} B-\frac{1}{2} C^{\sigma\{a} \partial_{\sigma} C^{b\}}\right) \mu_{b}, \\
\delta C^{\sigma a} & =\left(\varepsilon^{a b} B^{\sigma}-\frac{1}{2} C^{\lambda\{a} \partial_{\lambda} C^{\sigma b\}}\right) \mu_{b} .
\end{aligned}
$$

which, consequently, realize the transformations of extended BRST symmetry in terms of the variables $\phi^{A}$ of the complete configuration space. Again, the effective action, using the corresponding (anti)BRST operators, can be written in a compact form:

$$
S_{\mathrm{eff}}=S_{\mathrm{cl}}+\frac{1}{2} \varepsilon_{a b} s^{b} s^{a} F\left(e_{\mu}^{i}, \omega_{\mu}\right),
$$

which coincides with the effective action in the $S p(2)$-covariant approach [43]. (In comparision with that reference some misprints have been removed in eqs. (4.7)). 


\section{Conclusion}

In this paper we have exemplified the method of modified triplectic quantization [6] on the basis of several gauge theory models. Thus, we have considered the model [17] of non-abelian antisymmetric tensor field (Freedman-Townsend model), the model [18] of $W_{2}$-gravity, and the model [19] of two-dimensional gravity with dynamical torsion. For these models we have found explicit solutions of the generating equations that determine the (tree approximation of the) quantum action $W$ and the gauge-fixing functional $X$ in the framework of the modified triplectic formalism [6]. In the case of the 2-dimensional models we did not determine possible anomalies which occure if loop correction are taken into account.

The above solutions are expressed in terms of the variables $\phi^{A}, \phi_{A a}^{*}$ and $\pi^{A a}, \bar{\phi}_{A}$ anticanonically conjugated in the sense of the extended antibrackets [2, 6], as well as in terms of the additional variables $\lambda^{A}$ that serve to parametrize the gauge-fixing functional $X$. However, it should be remarked that by the special choice of both the action functional and of the gauge fixing functional triplecticity of the formalism is reduced, in fact, to the usual case of the $S p(2)$-covariant quantization.

Using the solutions for $S$ and $X$, we have obtained the vacuum functional and explicitly constructed the corresponding transformations [6] of extended BRST symmetry in terms of the anticanonically conjugated variables. Finally, we have obtained manifest $S p(2)$ symmetric expressions for the effective action $S_{\text {eff }}$ that result from integrating out the variables $\phi_{A a}^{*}, \pi^{A a}, \bar{\phi}_{A}$ and $\lambda^{A}$ in the functional integral, and we have constructed the corresponding transformations (1.6) of extended BRST symmetry in terms of the variables $\phi^{A}$ of the complete configuration space. In the case of the irreducible theories [18, 19], the resulting actions $S_{\text {eff }}$ have been represented in a compact form, expressing the gauge-fixed part in terms of (anti)BRST variations. In any case we finally obtained a $S p(2)$-symmetric action which is invariant under BRST and antiBRST transformations. In the case of irreducible theories we were able to write down the gauge fixing part in a very simple manner. In the case of the first-stage reducible FT model the situation occured much more difficult. Especially the dependence of the corresponding (anti)BRST transformation for the classical $H$-field on the gauge parameter $\alpha$ deserves further study.

\section{Acknowledgments}

The authors benefited from various discussions with D.M. Gitman. B.G. grateful acknowledges support from the German-Brasil exchange programmes DAAD and FAPESP, as well as the warm hospitality of the Institute of Mathematical Physics of the University of São Paulo, where this work was completed. The work of P.M.L. and P.Yu.M. was partially supported by the Russian Foundation for Basic Research (RFBR), project 99-02-16617, and the Russian Ministry of Education (Fundamental Sciences Grant, E003.3-461). The work of P.M.L. was supported by INTAS, grant 99-0590, as well as by the joint project of RFBR and Deutsche Forschungsgemeinschaft (DFG), 99-02-04022. P.M.L. also gratefully acknowledges the hospitality of NTZ at the Center of Advanced Study of Leipzig University.

\section{Appendix}

Here, we prove the representation $(2.8)-(2.10)$ for the vacuum functional in the Freedman-Townsend model (2.1) - (2.3). To this end, we consider the vacuum functional (1.4) with $S$ and $X$ given by eqs. (2.5) and (2.6), respectively. Integrating in eq. (1.4) 
over $\lambda^{A}$ and $\bar{\phi}_{A}$ leads to the replacement

$$
\bar{A}_{\mu}=\bar{B}_{\mu}=\bar{B}_{a}=\bar{C}_{a b}=0, \quad \bar{H}_{\mu \nu}=\frac{\alpha}{2} H_{\mu \nu}, \quad \bar{C}_{\mu a}=\beta \varepsilon_{a b} C_{\mu}^{b}
$$

Integrating over the variables $\pi^{A a}$ and the antifields $\phi_{A a}^{*}$, except $H_{\mu \nu a}^{*}$ and $C_{\mu a \mid b}^{*}$, with the subsequent replacements

$$
B_{\mu a}^{*}=C_{a \mid b c}^{*}=0
$$

leads to

$$
Z=\int d \phi d H_{\mu \nu a}^{*} d C_{\mu a \mid b}^{*} \exp \left\{\frac{i}{\hbar} \tilde{S}\left(\phi, H^{*}, C^{*}\right)\right\}
$$

with

$$
\begin{aligned}
\tilde{S} & =S_{\mathrm{cl}}+\int d^{4} x\left\{H_{\mu \nu a}^{*}\left(\mathcal{D}^{\mu} C^{\nu a}-\mathcal{D}^{\nu} C^{\mu a}\right)-\varepsilon^{a b} C_{\mu a \mid b}^{*} B^{\mu}-\frac{\alpha}{2} H_{\mu \nu}\left(\mathcal{D}^{\mu} B^{\nu}-\mathcal{D}^{\nu} B^{\mu}\right)\right. \\
& \left.+C_{\mu a \mid b}^{*} \mathcal{D}^{\mu} C^{a b}+\beta \varepsilon_{a b} C_{\mu}^{b} \mathcal{D}^{\mu} B^{a}+\frac{1}{2} \varepsilon^{\mu \nu \rho \sigma}\left(H_{\mu \nu a}^{*} \wedge H_{\rho \sigma b}^{*}\right) C^{a b}+\frac{\alpha}{4} \varepsilon^{\mu \nu \rho \sigma} H_{\mu \nu a}^{*}\left(H_{\rho \sigma} \wedge B^{a}\right)\right\} \\
& +\int d^{4} x\left\{\frac{1}{\alpha} H_{\mu \nu a}^{*} H_{b}^{* \mu \nu} \varepsilon^{a b}+\frac{1}{2 \beta} C_{\mu a \mid b}^{*} C_{c \mid d}^{* \mu} \varepsilon^{a c} \varepsilon^{b d}\right\},
\end{aligned}
$$

where $S_{\mathrm{cl}}$ is the classical action (2.1) of the Freedman-Townsend model.

Let us represent $Z$ in eq. (A.1) as

$$
Z=\int d \phi \exp \left\{\frac{i}{\hbar} S_{\mathrm{eff}}(\phi)\right\}
$$

where

$$
\exp \left\{\frac{i}{\hbar} S_{\mathrm{eff}}\right\}=\int d H_{\mu \nu a}^{*} d C_{\mu a \mid b}^{*} \exp \left\{\frac{i}{\hbar} \tilde{S}\left(\phi, H^{*}, C^{*}\right)\right\} .
$$

It is convenient to rewrite eq. (A.3) in the form

$$
\exp \left\{\frac{i}{\hbar} S_{\text {eff }}\right\}=C \cdot H \exp \left\{\frac{i}{\hbar}\left[S_{0}+\int d^{4} x\left(-\alpha B_{\mu \nu} \mathcal{D}^{\mu} B^{\nu}+\beta \varepsilon_{a b} C_{\mu}^{b} \mathcal{D}^{\mu} B^{a}\right)\right]\right\}
$$

with $C$ and $H$ being integrals over $C_{\mu a \mid b}^{*}$ and $H_{\mu \nu a}^{*}$, respectively,

$$
\begin{aligned}
C & =\int d C_{\mu a \mid b}^{*} \exp \left\{\frac{i}{\hbar} \int d^{4} x\left(C_{\mu a \mid b}^{*}\left(\mathcal{D}^{\mu} C^{a b}-\varepsilon^{a b} B^{\mu}\right)+\frac{1}{2 \beta} C_{\mu a \mid b}^{*} C_{c \mid d}^{* \mu} \varepsilon^{a c} \varepsilon^{b d}\right)\right\} \\
H & =\int d H_{\mu \nu a}^{*} \exp \left\{\frac{i}{\hbar} \int d^{4} x\left(H_{\mu \nu a}^{*} G^{\mu \nu a}+\frac{1}{\alpha} H_{\mu \nu a}^{*} H_{b}^{* \mu \nu} \varepsilon^{a b}+\frac{1}{2} \varepsilon^{\mu \nu \rho \sigma}\left(H_{\mu \nu a}^{*} \wedge H_{\rho \sigma b}^{*}\right) C^{a b}\right)\right\}
\end{aligned}
$$

where the object $G_{\mu \nu}^{a}$ is given by eq. (2.11).

The integral $C$ in eq. (A.5) is Gaussian and can be easily calculated,

$$
C=\exp \left\{-\frac{i}{\hbar} \int d^{4} x\left(\beta B_{\mu} B^{\mu}+\frac{\beta}{2} \varepsilon_{a b} \varepsilon_{c d}\left(\mathcal{D}_{\mu} C^{a c}\right)\left(\mathcal{D}^{\mu} C^{b d}\right)\right)\right\}
$$


In order to calculate $H$ in eq. (A.5), we use the decompositions

$$
\begin{aligned}
H_{\mu \nu a}^{*} & =\left(H_{o i a}^{*}, H_{i a}^{*}\right), H_{i a}^{*} \equiv \frac{1}{2} \varepsilon_{o i j k} H_{a}^{* j k}, \\
G_{\mu \nu}^{a} & =\left(G_{o i}^{a}, G_{i}^{a}\right), \quad G_{i}^{a} \equiv \frac{1}{2} \varepsilon_{o i j k} G^{j k a}
\end{aligned}
$$

and the following relations:

$$
\begin{aligned}
& H_{\mu \nu a}^{*} G^{\mu \nu a}=2\left(H_{o i a}^{*} G^{o i a}-H_{i a}^{*} G^{i a}\right), \\
& \varepsilon^{a b} H_{\mu \nu a}^{*} H_{b}^{* \mu \nu}=2 \varepsilon^{a b}\left(H_{o i a}^{*} H_{b}^{* o i}-H_{i a}^{*} B_{b}^{* i}\right), \\
& \frac{1}{2} \varepsilon^{\mu \nu \rho \sigma}\left(H_{\mu \nu a}^{*} \wedge H_{\rho \sigma b}^{*}\right) C^{a b}=4\left(H_{o i a}^{*} \wedge H_{b}^{* i}\right) C^{a b} .
\end{aligned}
$$

Integrating out the components $H_{i a}^{*}$ allows to represent $H$ in eq. (A.5) as

$$
\begin{aligned}
H & =\int d h_{i a}^{*} \exp \left\{\frac { i } { \hbar } \int d ^ { 4 } x \left(\frac{1}{2 \alpha} h_{i a}^{*} h_{b}^{* i} \varepsilon^{a b}+h_{i a}^{*} G^{o i a}\right.\right. \\
& \left.\left.-\frac{\alpha}{2} \varepsilon_{a b}\left(G_{i}^{a}-h_{i c}^{*} \wedge C^{a c}\right)\left(G^{i b}-h_{d}^{* i} \wedge C^{b d}\right)\right)\right\}
\end{aligned}
$$

where, for brevity, we denote $h_{i a}^{*} \equiv 2 H_{o i a}^{*}$.

Let us further rewrite $H$ in eq. (A.8) as

$$
H=\int d h_{i a}^{*} \exp \left\{\frac{i}{\hbar} \int d^{4} x\left(-\frac{\alpha}{2} \varepsilon_{a b} G_{i}^{a} G^{i b}+h_{i a}^{*} \bar{G}^{i a}+\frac{1}{2 \alpha} h_{i a}^{*} \mathcal{M}^{a b} h_{b}^{* i}\right)\right\},
$$

where

$$
\bar{G}_{i}^{a} \equiv G_{0 i}^{a}+\alpha \mathcal{C}_{b}^{a} G_{i}^{b},
$$

and the objects $\mathcal{C}_{b}^{a}, \mathcal{M}^{a b}$ are given by eqs. (2.12), (2.13). From the definitions (2.12) and (2.13) follow the algebraic properties

$$
\begin{gathered}
\left(\mathcal{C}_{b}^{a} F\right) G=-F\left(\mathcal{C}_{b}^{a} G\right), \quad \varepsilon^{a c} \mathcal{C}_{c}^{b}=-\mathcal{C}_{c}^{a} \varepsilon^{c b}, \quad \mathcal{C}_{a}^{c} \varepsilon_{c b}=-\varepsilon_{a c} \mathcal{C}_{b}^{c} \\
\mathcal{M}^{a c} \mathcal{C}_{c}^{b}=-\mathcal{C}_{c}^{a} \mathcal{M}^{c b}, \quad \mathcal{M}_{a c}^{-1} \mathcal{C}_{b}^{c}=-\mathcal{C}_{a}^{c} \mathcal{M}_{c b}^{-1}
\end{gathered}
$$

where $F \equiv F^{p}, G \equiv G^{p}$ are arbitrary objects carrying the gauge indices $p$, and the matrix $\mathcal{M}_{a b}^{-1}$ is the inverse of $\mathcal{M}^{a b}$ as defined in eq. (2.12).

Integrating in eq. (A.9) over $h_{i a}^{*}$ leads to

$$
H=\exp \left\{\frac{i}{\hbar} Y\right\} \int d h_{i a}^{*} \exp \left\{\frac{i}{2 \alpha \hbar} \int d^{4} x h_{i a}^{*} \mathcal{M}^{a b} h_{b}^{* i}\right\}
$$

where

$$
Y=\int d^{4} x\left\{\frac{\alpha}{2} \bar{G}_{i}^{a} \mathcal{M}_{a b}^{-1} \bar{G}^{i b}-\frac{\alpha}{2} G_{i}^{a} G^{i b} \varepsilon_{a b}\right\} \equiv \int d^{4} x \mathcal{Y}
$$

By virtue of the definition (A.10), the integrand $\mathcal{Y}$ in eq. (A.13) takes the form

$$
\begin{aligned}
\mathcal{Y}= & \frac{\alpha}{2} G_{0 i}^{a} \mathcal{M}_{a b}^{-1} G^{o i b}-\frac{\alpha}{2} \varepsilon_{a b} G_{i}^{a} G^{i b}+\frac{\alpha^{2}}{2} G_{0 i}^{a} \mathcal{M}_{a b}^{-1} \mathcal{C}_{c}^{b} G^{i c} \\
& +\frac{\alpha^{2}}{2} \mathcal{C}_{c}^{a} G_{i}^{c} \mathcal{M}_{a b}^{-1} G^{o i b}+\frac{\alpha^{3}}{2} \mathcal{C}_{c}^{a} G_{i}^{c} \mathcal{M}_{a b}^{-1} \mathcal{C}_{d}^{b} G^{i d}
\end{aligned}
$$


From the algebraic properties (A.11) it follows that

$$
\mathcal{C}_{c}^{a} G_{i}^{c} \mathcal{M}_{a b}^{-1} G^{o i b}=-G_{i}^{c} \mathcal{C}_{c}^{a} \mathcal{M}_{a b}^{-1} G^{o i b}=G_{i}^{c} \mathcal{M}_{c a}^{-1} \mathcal{C}_{b}^{a} G^{o i b}
$$

Similarly we have

$$
\mathcal{C}_{c}^{a} G_{i}^{c} \mathcal{M}_{a b}^{-1} \mathcal{C}_{d}^{b} G^{i d}=-G_{i}^{c} \mathcal{C}_{c}^{a} \mathcal{M}_{a b}^{-1} \mathcal{C}_{d}^{b} G^{i d}=G_{i}^{c} \mathcal{M}_{c a}^{-1} \mathcal{C}_{b}^{a} \mathcal{C}_{d}^{b} G^{i d}
$$

Note further that the combination $\mathcal{C}_{b}^{a} \mathcal{C}_{d}^{b}$ in eq. (A.16) can be represented as

$$
\mathcal{C}_{b}^{a} \mathcal{C}_{d}^{b}=\frac{1}{\alpha^{2}}\left(\mathcal{M}^{a b}-\varepsilon^{a b}\right) \varepsilon_{b d}
$$

Indeed, by virtue of the property $\mathcal{C}_{a}^{c} \varepsilon_{c b}=-\varepsilon_{a c} \mathcal{C}_{b}^{c}$ in eq. (A.11), we have

$$
\mathcal{C}_{c}^{a} \mathcal{C}_{b}^{c}=\mathcal{C}_{c}^{a} \mathcal{C}_{b}^{d} \delta_{d}^{c}=\mathcal{C}_{c}^{a} \mathcal{C}_{b}^{d} \varepsilon^{c p} \varepsilon_{p d}=\varepsilon^{c p} \mathcal{C}_{c}^{a} \mathcal{C}_{b}^{d} \varepsilon_{p d}=-\varepsilon^{c p} \mathcal{C}_{c}^{a} \mathcal{C}_{p}^{d} \varepsilon_{d b}
$$

which is equivalent to (A.17) with allowance for the definition $\mathcal{M}^{a b} \equiv \varepsilon^{a b}-\alpha^{2} \varepsilon^{c d} \mathcal{C}_{c}^{a} \mathcal{C}_{d}^{b}$.

Using eqs. (A.15) - (A.17) and the fact that $\mathcal{M}^{a c} \mathcal{M}_{c b}^{-1}=\delta_{b}^{a}$, we find that eq. (A.14) becomes

$$
\mathcal{Y}=\frac{\alpha}{2}\left(G_{0 i}^{a} \mathcal{M}_{a b}^{-1} G^{o i b}-G_{i}^{a} \mathcal{M}_{a b}^{-1} G^{i b}\right)+\frac{\alpha^{2}}{2}\left(G_{0 i}^{a} \mathcal{M}_{a b}^{-1} \mathcal{C}_{c}^{b} G^{i c}+G_{i}^{a} \mathcal{M}_{a b}^{-1} \mathcal{C}_{c}^{b} G^{0 i c}\right)
$$

which can be represented in a manifestly covariant form

$$
\mathcal{Y}=\frac{\alpha}{4} G_{\mu \nu}^{a} \mathcal{M}_{a b}^{-1} G^{\mu \nu b}+\frac{\alpha^{2}}{8} \varepsilon^{\mu \nu \rho \sigma} G_{\mu \nu}^{a} \mathcal{M}_{a b}^{-1} \mathcal{C}_{c}^{b} G_{\rho \sigma}^{c}
$$

From eq. (A.19) it follows that $H$ in eq. (A.12) is given by

$$
H=\Delta \exp \left\{\frac{\alpha i}{4 \hbar} \int d^{4} x G_{\mu \nu}^{a} \mathcal{M}_{a b}^{-1}\left(\frac{1}{2} \delta_{c}^{b}\left(\eta^{\mu \rho} \eta^{\nu \sigma}-\eta^{\mu \sigma} \eta^{\nu \rho}\right)+\frac{\alpha}{2} \varepsilon^{\mu \nu \rho \sigma} \mathcal{C}_{c}^{b}\right) G_{\rho \sigma}^{c}\right\}
$$

where $\Delta$ denotes the integral over $h_{i a}^{*}$ in eq. (A.12) and coincides with the corresponding object introduced in eq. (2.10).

Collecting eqs. (A.4), (A.6) and (A.20), we conclude that the vacuum functional (A.2) for the Freedman-Townsend model coincides with the representation (2.8) - 2.10).

\section{References}

[1] I.A. Batalin, P.M. Lavrov and I.V. Tyutin, J. Math. Phys. 31 (1990) 1487; 32 (1991) 532; 32 (1991) 2513.

[2] I.A. Batalin and R. Marnelius, Phys. Lett. B350 (1995) 44; Nucl.Phys. B465 (1996) 521 ;

I.A. Batalin, R. Marnelius and A.M. Semikhatov, Nucl.Phys. B446 (1995) 249.

[3] P.M. Lavrov, Phys. Lett. B366 (1996) 160; Theor. Math. Phys. 107 (1996) 602.

[4] B. Geyer and D. Mülsch, J. Math. Phys., 41 (2000) 7304.

[5] B. Geyer, P.M. Lavrov and D. Mülsch, J. Math. Phys. 40 (1999) 674; 40 (1999) 6189. 
[6] B. Geyer, D.M. Gitman and P.M. Lavrov, Mod. Phys. Lett. A14 (1999) 661; Theor. Mat. Fiz. 123 (2000) 476.

[7] C.M. Hull, Mod. Phys. Lett. A5 (1990) 1871.

[8] P.H. Damgaard and F. De Jonghe, Phys. Lett. 305B (1993) 59.

[9] P.H. Damgaard, F. De Jonghe and K. Bering K., Nucl. Phys. B455 (1995) 440.

[10] A. Nersessian and P.H. Damgaard, Phys. Lett. 355B (1995) 150.

[11] K. Bering, Mod. Phys. Lett. A11 (1996) 499.

[12] B. Geyer, P. Lavrov and A. Nersessian, "Poisson structure in BRST-antiBRST invariant Lagrangian formalism", hep-th/0104189.

[13] L. Tătaru and R. Tătar, Int. J. Mod. Phys. A13 (1998) 1981.

[14] C. Becchi, A. Rouet and R. Stora, Phys. Lett. B52 (1974) 344;

I.V. Tyutin, Gauge invariance in field theory and statistical physics in operator formalism, Lebedev Inst. preprint No. 39 (1975).

[15] G. Curci and R. Ferrari, Phys. Lett. B63 (1976) 91;

I. Ojima, Prog. Theor. Phys. Suppl. 64 (1979) 625.

[16] I.A. Batalin and G.A. Vilkovisky, Phys. Lett. B102 (1981) 27; Phys. Rev. D28 (1983) 2567.

[17] D.Z. Freedman and P.K. Townsend, Nucl. Phys. B177 (1981) 28.

[18] A.M. Polyakov, Mod. Phys. Lett. A2 (1987) 893.

[19] M.O. Katanaev and I.V. Volovich, Ann. Phys. 197 (1990) 1.

[20] W. Troost, P. van Nieuwenhuizen and A. Van Proeyen, Nucl. Phys. B333 (1990) 727.

[21] F. de Jonghe, R. Siebelink and W. Troost, Phys. Lett. B306 (1993) 295.

[22] S. Vandoren and A. Van Proeyen, Nucl. Phys. B411 (1994) 257.

[23] E. Witten, Nucl. Phys. B268 (1986) 253.

[24] S.P. De Alwis, M.T. Grisaru and L. Mezincescu, Phys. Lett. B190 (1987) 122;

A.A. Slavnov and S.A. Frolov, Teor. Mat. Fiz. 75 (1988) 201;

C. Battle and J. Gomis, Phys. Rev. D38 (1988) 1169.

[25] P.M. Lavrov and P.Yu. Moshin, Theor. Math. Phys. 104 (1995) 1098.

[26] T.E. Clark, C.H. Lee and S.T. Love, Nucl. Phys. B308 (1988) 379.

[27] P.M. Lavrov and I.V. Tyutin, Sov. J. Nucl. Phys. 50 (1989) 912.

[28] J. Thierry-Mieg, Nucl. Phys. B335 (1990) 334.

[29] G. Barnich, R. Constantinescu and P. Gregoire, Phys. Lett. B 293 (1992) 353.

[30] M. Abud, L. Cappiello and J.-P. Ader, Int. J. Mod. Phys. A 10 (1995) 1577. 
[31] E.M.C. Abreu and N.R.F. Braga, Phys. Rev. D54 (1996) 4080.

[32] F. De Jonghe, The Batalin-Vilkovisky Lagrangian quantisation scheme with applications to the study of anomalies in gauge theories, hep-th/9403143.

[33] N.R.F. Braga and C.F.L. Godinho, Phys.Rev. D61 (2000) 125003.

[34] M.O. Katanaev and I.V. Volovich, Phys. Lett. B175 (1986) 413.

[35] M.O. Katanaev, J. Math. Phys. 31 (1990) 882; 32 (1991) 2483; 34 (1993) 700.

[36] W. Kummer and D.J. Schwarz, Phys. Rev. D45 (1992) 3628.

[37] P. Schaller and T. Strobl, Lecture Notes in Physics 469 (1996) 321; Poisson-sigmamodels: a generalization of 2-d gravity Yang-Mills-systems, hep-th/9411163.

[38] R. Marnelius, Nucl. Phys. B211 (1983) 14; C. Teitelboim, Phys. Lett. B126 (1983) 41;

T. Banks and L. Susskind, Int. J. Theor. Phys. 23 (1984) 475;

R. Jackiw, Nucl. Phys. B252 (1985) 343;

I. M. Lichtzier and S. D. Odintsov, Mod. Phys. Lett. A6 (1991) 1953.

[39] N. Ikeda and K. Izawa, Prog. Theor. Phys. 89 (1993) 223.

[40] T. Strobl, Int. J. Mat. Phys. A8 (1993) 1383;

[41] P. Schaller and T. Strobl, Class. Quant. Grav. 11 (1994) 331; F. Haider and W. Kummer, Int. J. Mod. Phys. A9 (1994) 207.

[42] W. Kummer and D.J. Schwarz, Nucl. Phys. B382 (1992) 171.

[43] P.M. Lavrov and P.Yu. Moshin, Theor. Math. Phys. 114 (1998) 313, Teor. Mat. Fiz. 114 (1999) 399; Class. Quant. Grav. 16 (1999) 2247. 